Pathologe 2017 - 38 (Suppl 2):S238 https://doi.org/10.1007/s00292-017-0385-7

Online publiziert: 24. Oktober 2017

C) Springer Medizin Verlag GmbH 2017

CrossMark

\author{
A. Agaimy \\ Institut für Pathologie, Universitätsklinikum Erlangen, Erlangen, Deutschland
}

\section{Bericht des Vorsitzenden der Arbeitsgruppe für Kopf-/ Halspathologie der Deutschen Gesellschaft für Pathologie}

\section{Jahresaktivitäten 2016/2017}

Seit der strukturellen Neuausrichtung und Umbenennung der Arbeitsgruppe von AG Oralpathologie in AG Kopf-/ Halspathologie auf der Jahrestagung der Deutschen Gesellschaft für Pathologie (DGP) 2014 hat sich die AG intensiv für unser Teilgebiet eingesetzt und durch eine starke Unterstützung der DGP einen großen Erfolg erlangt.

Ich möchte mich als Vorsitzender der AG in diesem Zusammenhang - auch im Namen aller Mitglieder der Arbeitsgruppe - beim Vorstand der DGP bedanken. Unter oben genannter Unterstützung durch die DGP und unter Mitwirkung meines Vorgängers Herrn Prof. Wilko Weichert und meines Stellvertreters Herrn Prof. Sven Perner hat sich die AG zu einer der erfolgreichsten AGs entwickelt.

Höhepunkt der AG-Erfolge war in diesem Jahr sicherlich die Tatsache, dass die Kopf-Hals-Pathologie zum Hauptthema der diesjährigen 101. Jahrestagung der Deutschen Gesellschaft für Pathologie in Erlangen geworden ist, bei der neben einem sehr umfangreichen Beitrag aus der Poster-Session sowie freien Forschungsbeiträgen im Rahmen des Hauptprogramms und der AG-Sitzung am Donnerstagnachmittag auch drei Hauptveranstaltungen stattfanden. Zum einen wurden die neuen Entwicklungen in der Kopf-Hals-Pathologie in einer gesonderten Session zu „emerging entities in head and neck pathology" abgehandelt, dazu waren mehrere renommierte internationale Referenten unter starker
Einbeziehung der „Working Group Head and Neck Pathology“ der ESP wie Prof. Michal Michal, Prof. Alena Skalova und Prof. Alessandro Franchi eingeladen. Zum anderen wurden die neuen Entwicklungen der molekularen Pathologie der Kopf-Hals-Region in einer hierfür gesonderten Sitzung abgehandelt - auch hier wurden sehr prominente Referenten aus den USA und anderen Ländern eingeladen. Ein Key-note-Vortrag war dem Thema „molekulare Pathologie der Kopf-Hals-Region“ gewidmet. Ferner wurde eine klinische Session zum Thema „klinische Kopf-Hals-Pathologie“ einschließlich der molekularen Aspekte in einer hierfür gesonderten Sitzung unter Einbeziehung klinischer Kollegen beleuchtet.

Zum ersten Mal wurde im Rahmen der DGP-Tagung die enge Beziehung der Kopf-Hals-Pathologie zur Neuropathologie in zwei besonderen Sitzungen herausgearbeitet: eine Sitzung zum Thema „skull invaders“ und ein gemeinsames IAP-Seminar zur Pathologie der Schädelbasis zusammen mit Kollegen aus der Neuropathologie und der Ophthalmopathologie organisiert. Dieses fand eine hohe Akzeptanz, wie es den zahlreichen positiven Rückmeldungen zu entnehmen war. An dieser Stelle möchte ich mich auch im Namen der AG-Mitglieder ganz besonders bei Herrn Prof. Arndt Hartmann, dem Tagungspräsidenten der 101. Jahrestagung der DGP, bedanken.

Einen weiteren Höhepunkt der Erfolge der Kopf-Hals-Gruppe stellt das be- vorstehende IAP-Symposium $2018 \mathrm{mit}$ den Neuigkeiten der WHO-Klassifikation von Kopf-Hals-Tumoren als Hauptthema des Ganztagsseminars im kommenden Februar dar.

Wir hoffen für das kommende Jahr durch die genannten Aktivitäten und die kontinuierliche, konsequente Fortführung des eingeschlagenen Weges, noch weitere Kollegen aus der Pathologie für unser stets wachsendes und in einigen Aspekten noch „unterforschtes“ Teilgebiet der modernen Pathologie interessieren und gewinnen zu können. Nur so können wir die Grundlage für einen zunehmend erfolgreichen Beitrag der deutschen Pathologen in der Erforschung und Fortentwickelung der KopfHals-Pathologie in der nächsten Dekade legen.

\section{Korrespondenzadresse}

Prof. Dr. A. Agaimy

Institut für Pathologie, Universitätsklinikum Erlangen

Krankenhausstraße 8-10, 91054 Erlangen,

Deutschland

abbas.agaimy@uk-erlangen.de

\section{Einhaltung ethischer Richtlinien}

Interessenkonflikt. A. Agaimy gibt an, dass kein Interessenkonflikt besteht.

Dieser Beitrag beinhaltet keine vom Autor durchgeführten Studien an Menschen oder Tieren.

The supplement containing this article is not sponsored by industry. 